NASA Technical Memorandum 107288

AIAA-96-2550

\title{
Minimum Weight Design of a Generic Axisymmetric Inlet
}

Shari-Beth Nadell

Lewis Research Center

Cleveland, Ohio

Prepared for the

32nd Joint Propulsion Conference

cosponsored by AIAA, ASME, SAE, and ASEE

Lake Buena Vista, Florida, July 1-3, 1996

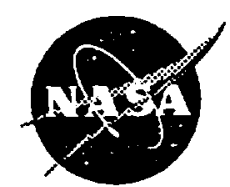

National Aeronautics and

Space Administration 



\title{
MINIMUM WEIGHT DESIGN OF A \\ GENERIC AXISYMMETRIC INLET
}

\author{
Shari-Beth Nadell* \\ NASA Lewis Research Center, Cleveland, Ohio 44135
}

\begin{abstract}
A new minimum weight design method for highspeed axisymmetric inlets was demonstrated on a generic inlet. The method uses Classical Beam Theory and shell buckling to determine the minimum required equivalent isotropic thickness for a stiffened shell based on prescribed structural design requirements and load conditions. The optimum spacing and equivalent isotropic thickness of ring frame supports are computed to prevent buckling. The method thus develops a preliminary structural design for the inlet and computes the structural weight. Finite element analyses were performed on the resulting inlet design to evaluate the analytical results. Comparisons between the analytical and finite element stresses and deflections identified areas needing improvement in the analytical method. The addition of the deflection due to shear and a torsional buckling failure mode to the new method brought its results in line with those from the finite element analyses. Final validation of the new method will be made using data from actual inlets.

\section{Nomenclature}

E modulus of elasticity, $\mathrm{lb} / \mathrm{in}^{2}$

$\mathrm{F}_{\text {Scr }}$ design allowable shear stress to prevent torsional buckling, $\mathrm{lb} / \mathrm{in}^{2}$

$\mathrm{G}$ shear modulus of elasticity, lb/in ${ }^{2}$

$\mathbf{K}_{\text {non-opt }}$ non-optimum weight factor

$\mathrm{L}$ shell length for torsional buckling, in

n load factor

$N_{x y} \quad$ shear stress resultant, $l b /$ in

$r, R$ shell radius, in

$S$ surface area, in ${ }^{2}$

$t$ thickness; equivalent isotropic thickness, in

$t_{\text {buck }}$ minimum equivalent isotropic thickness of shell to prevent radial buckling, in

$\mathrm{t}_{\text {comp }}$ minimum equivalent isotropic thickness of shell to prevent compression failure, in

$t_{\text {frame }}$ minimum equivalent isotropic thickness of frame, in
\end{abstract}

\author{
$t_{\text {long }}$ minimum equivalent isotropic thickness of shell \\ to prevent longitudinal buckling, in \\ $t_{m g} \quad$ minimum gage thickness of the shell, in \\ $t_{\min }$ minimum equivalent isotropic thickness of shell, \\ in \\ $\mathrm{t}_{\text {shear }}$ minimum equivalent isotropic thickness of shell \\ to prevent shear failure, in \\ $t_{\text {tension }}$ minimum equivalent isotropic thickness of shell \\ to prevent tensile failure, in \\ $t_{\text {torsion }}$ minimum equivalent isotropic thickness of shell \\ to prevent torsional buckling, in \\ W weight, lb \\ $W_{\text {seg }}$ segment weight, lb \\ $W_{\text {str }}$ structural weight, lb \\ $\mathrm{x} \quad \mathrm{x}$ coordinate value, in \\ $\gamma \quad$ unit shear strain \\ $\delta \quad$ bending deflection, in \\ $\Delta \quad$ shear deflection, in \\ $\pi \quad 3.14159$ \\ $\rho \quad$ material density, $l b / \mathrm{in}^{3}$ \\ $\sigma_{\mathrm{x}} \quad$ bending stress, $\mathrm{lb} / \mathrm{in}^{2}$ \\ $\sigma_{y} \quad$ hoop stress, $1 \mathrm{~b} / \mathrm{in}^{2}$ \\ $\tau \quad$ shear stress, $1 \mathrm{~b} / \mathrm{in}^{2}$ \\ Subscripts \\ $\mathrm{i}$ analysis station counter \\ $\mathrm{k}$ sum variable

\section{Introduction} \\ Current aeropropulsion systems studies are \\ focusing on advanced concepts that very often do not \\ draw on current and past design practices enough to \\ permit the use of empirically based design and weight \\ analysis methods. New methods for the conceptual \\ design and analysis of advanced engine components \\ must therefore be developed and tested. These new \\ methods can also provide a bridge between conceptual \\ level design and more detailed, complex preliminary \\ design activities.
}

* Aerospace Engineer, Member AIAA 
To address this issue in the area of high-speed inlet design and weight analysis, a minimum weight design method was developed based on analytical principles. ${ }^{1}$ The analytical method requires as input only information that is available from conceptual flowpath analysis. Using this information, the analytical method, called the Axisymmetric Inlet Minimum Weight Design Method, or AXIDES, develops an initial structural design and computes the structural weight of the inlet. The method assumes stiffened shell construction of the inlet structure. Various types of stiffener cross-sections can be specified, including truss-core honeycomb construction. Classical Beam Theory and shell radial buckling analysis due to an external load are employed to determine the minimum required equivalent isotropic thickness for the stiffened shell based on prescribed structural design requirements and load conditions. The optimum spacing and equivalent isotropic thickness of ring frame supports are computed to prevent panel buckling. Additional weight for systems is added to the structural weight, resulting in an estimate for the total weight of the inlet.

The AXIDES method was demonstrated using a generic Mach 2.4 axisymmetric inlet. The resulting minimum weight inlet design was then evaluated using finite element analyses (FEA). The goals of the evaluation included identifying possible deficiencies in the new analytical method as well as measuring its accuracy. Any deficiencies were then corrected by modifying the AXIDES method.

This paper gives a brief description of the minimum weight design method and then discusses the application of the method to a generic Mach 2.4 axisymmetric inlet. The development of finite element models based on the analytical design results is presented along with a comparison of the deflection and stress results between the two analyses. Modifications made to the analytical method as a result of the FEA evaluation are described.

\section{Design Method Summary}

The general strategy used in the Axisymmetric Inlet Minimum Weight Design Method is similar to that used by Ardema for the minimum weight design of arbitrary fuselage bodies. ${ }^{2}$ In essence, the method determines the minimum "smeared" thickness, which is the minimum equivalent isotropic thickness. of a stiffened panel structure required to prevent failure. The weight of the structure is then calculated based on this smeared thickness value. A detailed description of the AXIDES method can be found in reference 1. The method is summarized below.
The AXIDES method assumes stiffened shell construction, i.e., shells or panels stiffened by longitudinal members and ring frames (Figure 1). Nine different structural concepts are available for the design The cross-section of the ring frames is assumed to be $Z$ shaped for all of the structural concepts. To simplify the inlet structure, it is divided into three components: centerbody, internal cowl, and external cowl (Figure 2). It is then assumed that each of these structures can be designed and analyzed separately from the others. While there is no question that these structures actually do interact, this simplification was felt to be reasonable for conceptual level design.

The first step in the minimum weight design process is the definition of the inlet geometry. An initial geometric definition of the inlet flowpath is obtained from conceptual level performance analyses. This geometry is refined using parametric cubic curves to produce a smooth surface that is integrated to find surface areas, volumes, and center of gravity locations. A loads analysis is then performed at equally spaced stations along the length of each inlet component. Various combinations of internal and external loads, including inertial loads, internal pressures, and aerodynamic loads, are considered. The AXIDES method requires that normal operating and hammershock pressures be supplied at each geometric coordinate of the inlet flowpath. Hammershock pressures result from dynamic flow distortion that causes the engine to surge. This results in a shock wave that moves forward out of the inlet, leaving behind pressures that are significantly higher than the inlet pressure before the distortion. ${ }^{3}$ External pressures are likewise required at each geometric coordinate defining the external cowl structure.

Eight different design load cases incorporating the inlet pressures and loads are used to determine the maximum loads to which the structure will be designed. These are: landing, yaw maneuver, lateral load with nose left yawing moment, vertical load with nose down pitching moment, wind gust, asymmetric hammershock, normal operating pressure, and axisymmetric hammershock. Each is assumed to occur independent of the others. The maximum loads for all of the design load cases are based on ultimate load requirements. Each design load case produces some combination of bending, shear, axial, and radial loads. For the calculation of bending and shear loads, the structure is assumed to act like a cantilever beam, fixed at a location approximating the centerline of the support struts that would be present in an actual inlet (Figure 2). Computation of axial and radial loads assumes that the 
structure acts like a thin-walled cylindrical shell. The final maximum loads at each analysis station are chosen by comparing those computed for each design load case. The final load distribution is therefore not based solely on one load condition, but on a combination of those that produce the most severe loads that the structure will be subject to during all levels of operation.

Assuming elastic behavior for all structures, the minimum allowable smeared thickness of the shell/longitudinal stiffeners $\left(t_{\text {min }}\right)$ is computed at each station based on the maximum loads applied at that station. Five possible failure modes are considered in the calculation of $t_{\min }$. These are tension, compression without buckling, shear, longitudinal buckling, and radial buckling. The last of these is only considered in the design of the inlet centerbody, which is subject to external pressure loads. Maximum stress failure theory is applied for all failure modes. A minimum gage constraint, due to either manufacturing considerations or Foreign Object Damage (FOD) requirements, is included as an additional design requirement. Six values for $t_{\min }$ are therefore computed at each analysis station, one for each failure mode and one for minimum gage. The final minimum smeared thickness at each analysis station is determined by comparing those from each failure mode, or

$$
t_{\text {min }}=\max \left(t_{\text {tension }}, t_{\text {comp }}, t_{\text {long }}, t_{\text {shear }}, t_{\text {buck }}, t_{m g}\right)
$$

An iterative process is used to determine the optimum spacing and smeared thickness of the ring frames; alternatively, the frame spacing may be input to the method, and the appropriate frame smeared thickness $\left(t_{\text {frame }}\right)$ will then be computed. The thickness and spacing of the ring frames are based on the Shanley criterion. ${ }^{4}$ This assumes that the frames act as elastic supports for a wide column with an equal probability of general instability or local buckling failure. The spacing and minimum equivalent thickness of the frames is then based on panel failure due to buckling, since this is the simpler approach of the two.

The total structural weight of each inlet component is found by multiplying $t_{\min }$ and $t_{\text {frame }}$ by the material density and surface area of each analysis segment:

$$
W_{s t r}=\left[\pi \sum\left(\rho t_{\min }+\rho t_{\text {frame }}\right) S\right]\left(1+K_{\text {non-opt }}\right)
$$

where $K_{\text {non-opt }}$ is a non-optimum weight factor that is applied to the structural weight of the inlet to account for structural items such as fasteners and bolts, extra weld material, uniform gages, etc., that are not modeled in the structural analysis. This factor also corrects for some of the inaccuracies introduced by the simplifying assumptions made during the design process. The total structural weight of the inlet is the sum of the weight of the centerbody, internal cowl, and external cowl structures. Finally, additional weight is added to account for inlet actuators, controls, and bypass systems. This weight is interpolated from data used in the INSTAL program. ${ }^{5}$

The last action performed by the AXIDES program is the computation of the deflections, using the Moment-Area method ${ }^{6}$, of the internal surfaces of the inlet. This is important because the optimum performance of a high-speed inlet is dependent on maintaining the proper throat area and internal flowpath geometry during normal inlet operation. Similar to the inlet design, the inlet components are assumed to act as cantilever beams for the deflection calculations. Limit loads due to normal inlet operation were used for these calculations.

\section{Generic Inlet Description}

The AXIDES program was demonstrated using a generic Mach 2.4 axisymmetric inlet generated in-house at the NASA Lewis Research Center. The total length of the inlet (Figure 3), from centerbody tip to compressor face, is 169.48 in, and the cowl lip radius is 29.2 in. The inlet normal operating and hammershock pressures are plotted in Figure 4 along with the external pressure assumed to act on all external surfaces.

The inlet was designed using Titanium alloy Ti$6 \mathrm{Al}-4 \mathrm{~V}$. The material properties can be found in reference 7. Since the AXIDES program does not perform a thermal stress analysis, the effect of high temperatures on the inlet structure is introduced by degrading the material properties so they are consistent with the approximate continuous operating temperature on a standard day for a Mach 2.4 inlet.

The structural concept used in AXIDES for the generic inlet was Z-stiffened shell with frames designed for best buckling behavior. A fixed frame spacing of 10 in was specified. This value was chosen based on a trade study that compared inlet weight with frame spacing. ${ }^{8}$

\section{Analytical Design Results}

The total weight of the generic inlet, as computed by the AXIDES method, was $2773.79 \mathrm{lb}$. The inlet 
centerbody, internal cowl, and external cowl weights were $645.26 \mathrm{lb}, 413.00 \mathrm{lb}$, and $454.96 \mathrm{lb}$, respectively. Twenty percent of these weights were due to the nonoptimum weight penalty. The inlet systems accounted for $1260.58 \mathrm{lb}$ of the total inlet weight.

The minimum equivalent shell thicknesses for the inlet centerbody, internal cowl, and external cowl are shown in Figure 5. FOD requirements dictated the value of $t_{\min }$ at the forward part of the centerbody as well as at the cowl lip. The critical failure mode along most of the centerbody was radial buckling due to axisymmetric hammershock pressure loads. The internal cowl structure consisted of both minimum gage and shear critical equivalent shells. The structure for the external cowl was designed entirely by minimum gage constraints. The unit weight of all three inlet components is shown in Figure 6. All frame structures were designed based on minimum gage constraints, indicating the possibility that the Z-shaped cross-section may not have been optimum for this inlet design.

The maximum deflections of the centerbody and internal cowl occurred at the centerbody tip ( $x=0.0$ in) and the cowl lip (Figures 7 and 8). The maximum deflection of the centerbody was -.01617 in; the maximum deflection of the internal cowl was -.00176 in. Both of these values fell within the acceptable deflection limits as defined in the AXIDES program and in reference 9.

\section{Finite Element Evaluation}

\section{Finite Element Analysis}

The minimum weight structural design of the generic axisymmetric inlet developed using AXIDES was used to generate three models for finite element analysis. One model was created for each inlet component. These models were used to evaluate the stresses and deflections predicted by the analytical minimum weight design method for four of the design load cases. The MSC/PATRAN pre- and postprocessor ${ }^{10}$ was used to generate the finite element models and evaluate the FEA results. The GENESIS finite element analysis program ${ }^{11}$ was used to perform linear static analyses for each load case described below.

The AXIDES method assumes that the smeared shell structure carries all of the primary loads, with the ring frames providing stability. The structure modeled for the FEA analysis therefore only represented the equivalent shell structure from the analytical method. Nonstructural mass, including the weight of the ring frames and the non-optimum shell and frame weights, was included on the element property cards (PSHELL cards). Therefore, the correct inertial load was applied to the structure. QUAD4 two-dimensional elements were used to model the equivalent shell structure. The inlet centerbody was modeled using 2503 QUAD4 elements. The internal and external cowls were modeled using 1392 and 1624 QUAD4 elements, respectively. The finite element models for the inlet centerbody, internal cowl, and external cowl are shown in Figure 9. Symmetric boundary conditions were used to reduce the size of the models. Fixed boundary conditions were applied at the aft nodes of each model to mimic the fixed cantilever end and to prevent warping of the final cross-section. These fixed boundary conditions are comparable to a rigid inlet mount.

The four design load cases, representative of the different types of loading, chosen for use in the method evaluation are the normal operating limit pressure load case, the landing load case, the axisymmetric hammershock load case, and the asymmetric hammershock load case. Each of these four load cases included the inertial load of the structure. This was modeled using the gravitational (GRAV) load card. Pressure loads were modeled using the PLOAD2 card. GENESIS does not support varying pressure loads across an individual element. Therefore, the pressure variations along the inlet axis were applied as a step function, with the changes occurring at each analysis station coordinate. This introduces small stress discontinuities across the nodes located at each analysis station.

\section{Comparison of FEA and AXIDES Results}

The evaluation of the AXIDES method consisted not only of comparing the analytical data with that from the finite element analyses, but also of identifying deficiencies in the analytical method, and improving the method to eliminate or reduce the impact of these deficiencies. Selected AXIDES stress and deflection results were compared with those obtained from the finite element analyses, as described below. Problem areas and the resulting modifications are discussed.

The analytical bending and hoop stresses for the individual load cases were computed using the AXIDES minimum weight design results. The finite element deflection results were recovered at the nodes in the $x-z$ plane $(y=0)$. Finite element stress results were recovered for the QUAD4 elements located at the top $(y>0, z=0)$ and bottom $(y<0, z=0)$ of the models. Assuming beam behavior, these locations should give 
the maximum tensile and compressive stresses in the model. It was assumed that the compressive bending stresses would have greater impact on the inlet design than the tensile bending stresses; therefore, only the compressive bending stresses, or those along the bottom of the structure, are compared. GENESIS produces stress results at the top (Z1) and bottom (Z2) faces of the QUAD4 plate elements, where the top face is in the positive direction of the normal vector of the element. For all three finite element models, the positive normal vector was in the negative radial direction. Both $Z I$ and $Z 2$ results are given in the figures presenting FEA stress results.

Deflections $(\delta)$ caused by limit normal operating pressure and gravitational loads were compared for the inlet centerbody and internal cowl structures. Bending stresses $\left(\sigma_{x}\right)$ due to landing gravitational loads, bending and hoop stresses $\left(\sigma_{y}\right)$ resulting from axisymmetric hammershock pressures and gravitational loads, and bending stresses caused by asymmetric hammershock and gravitational loads were compared for all three inlet components.

\section{Limit Normal Operating Deflection}

\section{Bending Deflection}

The analytical and FEA centerbody deflection in the $y$-direction caused by the limit normal operating inertial load are compared in Figure 7. The maximum deflection from AXIDES, -0.01617 in, was approximately 11.6 percent less than the -0.0183 in obtained using the GENESIS finite element program. This is good agreement given the assumptions made in the analytical method.

A comparison of the deflection results for the internal cowl structure can be seen in Figure 8 . The maximum deflection obtained from AXIDES was -0.00176 in, while that obtained from GENESIS was -0.00287 in. The analytical result is approximately 38.7 percent less than the finite element result. One possible explanation for this difference is the influence of the shear loads on the deflection of the structure. In general, the deflection due to shear is small for beams with large length to diameter ratio. However, as the length to diameter $(L D)$ ratio becomes small (the average length to diameter of the generic axisymmetric inlet cowl is approximately 1.98), the deflection due to shear becomes a larger part of the overall beam deflection. ${ }^{12}$ The computation of the deflection due to shear for the inlet is discussed in the following section.

\section{Shear Deflection}

The deflection due to shear was computed for the inlet structure using the following simplified method. The only load considered for this load case is the inertial load of the structure. It was assumed that the weight of each shell segment, where the length of one segment was equal to the distance between analysis stations, was distributed uniformly over the length of the segment. Furthermore, it was assumed that the weight of the segment acted at the forward edge of the segment, instead of at the center of gravity location of the segment. Assuming that

$$
\Delta=\gamma x=\frac{\tau x}{G}
$$

the maximum shear stress for a hollow circular crosssection is given by

$$
\tau=\frac{W n(t+r)}{\pi r^{2} t}
$$

Starting at the last (aft) segment of the shell and moving forward, the shear deflection at each analysis station was defined as the sum of the shear deflection of the previous station plus the shear deflection at the current station due to all of the segment weights ahead of that station:

$$
\Delta_{i}=\Delta_{i+1}+\left[\sum_{k=1}^{i} W_{s e g} n\right] \frac{\left(t_{m i n}+r_{i}\right)}{\pi r_{i}^{2} t_{\min }} \frac{\left(x_{i}-x_{i+1}\right)}{G}
$$

The deflections due to shear computed from Equation (5) for the inlet centerbody and internal cowl are presented in Figures 7 and 8, respectively. As seen in Figure 7, the addition of the shear deflection to the bending deflection gave a modest improvement in the analytical results for the inlet centerbody, which were already within 12 percent of the FEA results. The total maximum centerbody deflection increased from -.01617 in to -.01706 in. This new value is 6.8 percent lower than that computed by GENESIS. The addition of the shear deflection to the internal cowl bending deflection (Figure 8) decreased the difference between the AXIDES and FEA results from 38.7 percent to 21.6 percent. The new value of the total maximum internal cowl deflection was -.00225 in.

Though the addition of the shear deflection improved the comparison between the analytical and FEA analyses for this load case, Figure 8 shows that 
there is still a considerable difference in the internal cowl deflections. A correction factor, whose value is given as 2.0 for hollow cylindrical cross-sections, is applied in the literature when computing shear deflection. ${ }^{12}$ Applying this factor to the shear deflection of the internal cowl and adding the bending deflection gives the total deflection results shown in Figure 8. As can be seen, the maximum deflection, and the shape of the deflection curve, is much closer to the FEA results with the correction factor included. This maximum deflection is -.00275 in, 4.2 percent less than the finite element value. Increasing the correction factor to 2.25 gives almost exact agreement between the analytical and FEA results, with a maximum deflection of -.00287 in (Figure 8, " $\mathrm{B}+(\mathrm{S} * 2.25)$ ").

The results of similar increases in the inlet centerbody shear deflection are shown in Figure 7. Multiplying the centerbody shear deflection by 2.0 results in a maximum deflection of -.01797 in, only 1.8 percent less than that for the FEA model, whereas multiplying by 2.25 gives almost exactly the same results as the FEA, with a maximum deflection of -.0182 in.

Based on the preceding discussion, the deflection analysis of the AXIDES method was modified to include the deflection due to shear multiplied by a correction factor of 2.25 . The new method can thus predict the deflections of the inlet centerbody and internal cowl more accurately. Comparison with predefined deflection limits on the inlet will lead to better information for the user of the method regarding the need to redesign the inlet geometry.

\section{$\underline{\text { Landing Bending Stress }}$}

The landing load case is a pure bending situation, including only the inertial load of the structure. A comparison of the AXIDES and GENESIS bending stresses for the centerbody is shown in Figure 10. Good agreement between the results, like that seen in Figure 10 , is also obtained for the internal and external cowl structures. The influence of the fixed boundary conditions on the finite element model can be seen at the aft end ( $x=169.48$ in), where the FEA bending stresses vary suddenly from the expected distribution. This is strictly a result of the finite element method and does not reflect a real, physical condition.

\section{Axisymmetric Hammershock Bending and Hoop} $\underline{\text { Stresses }}$

The analytical and finite element hoop stresses caused by the axisymmetric hammershock pressure loads compare well with one another, as seen for the internal cowl in Figure 11. The effect of the FEA aft fixed boundary conditions are once again evident for all components.

Comparisons of the bending stresses for this same load case show a large difference between the analytical and FEA results. The bending stresses for the internal cowl are shown in Figure 12. A closer look at the FEA models indicates that local deformation of the QUAD4 elements due to the axisymmetric pressure loads are the major contributor to the bending stresses seen in the FEA results. The magnitudes of these local deformations are small, and the Von Mises stresses are much lower than the material yield stress. These local deformations are therefore not causing failure of the structure. They are a result of the way in which the FEA model was constructed, using equivalent thickness shells, and are, therefore, not necessarily indicative of the stresses in an actual inlet, which would be constructed using discrete structural elements. Comparing the finite element bending stresses due to only the inertia load with the analytical results shows good agreement similar to that found for the landing load case. No modifications to the computation of the bending stresses in AXIDES for this load case were deemed necessary due to the influence of the model technique on the results.

\section{Asymmetric Hammershock Bending Stress}

\section{Linear Static Analysis}

Comparison of the bending stresses computed for the inlet centerbody using AXIDES and GENESIS for the asymmetric hammershock load case indicates fairly good agreement, though not as close as the previous two bending load cases. Those for the internal and external cowls, however, do not show good agreement, as seen for the internal cowl in Figure 13. Furthermore, the bending stress plot for the internal cowl (Figure 14), and similarly for the external cowl, did not show the expected distribution with the largest tensile and compressive stresses at the top and bottom of the structure, respectively. For example, Figure 14 shows that the largest stresses in the internal cowl occur at approximately 65 and 120 degrees around the circumference of the shell.

The maximum FEA deflections of the internal and external cowl structures for the asymmetric hammershock load case were 36.6 and 17.5 in, respectively. The finite element linear static analysis is based on small displacement theory; the large maximum deflections seen in these results and the large stress 
variation around the circumference of the cowl shells were indications that these structures were not behaving in a linear fashion. Therefore, nonlinear buckling analyses were performed on these structures. This is presented in the following section. The maximum deflection of the centerbody structure for this load case was 2.2 in at the centerbody tip. The Von Mises stresses indicated no structural yield, and no further analysis on the centerbody was performed.

\section{Nonlinear Buckling Analysis}

Two changes were made to improve both the internal and external cowl FEA models before performing the nonlinear analyses. The first change involved increasing the mesh density from 1392 to 5568 elements for the internal cowl, and 1624 to 6496 elements for the external cowl. The second change made involved the application of the pressure loads to the elements. Whereas in the linear static analyses the pressure was applied uniformly over each element, resulting in a step function distribution along the length of the inlet, it was decided that improved accuracy could be obtained by varying the pressure linearly over each element, giving a smoother load distribution.

The GENESIS finite element program does not support varying pressure loads over an element. It was therefore necessary to switch to a different finite element analysis method. The NASTRAN finite element program ${ }^{13}$ was chosen. NASTRAN supports the same QUAD4 element as GENESIS. The pressures on the elements were applied using the PLOAD4 card, which varies the pressure linearly across the QUAD4 element. All boundary conditions were applied in the same manner as for the linear static analysis FEA models.

The nonlinear buckling analysis of the inlet cowl structures showed that the internal cowl was only able to carry 27.8 percent of the applied asymmetric hammershock pressure loads, and the external cowl could carry only 2.8 percent. At these percentages of the load, the shear stresses in shells reach the critical value for buckling. Figure 15 shows the buckled shape of the internal cowl. As seen in this figure, the buckling is a rippling in the aft portion of the shell, rather than an obvious rupture. The buckled area of the internal cowl can also be seen in the shear stress distribution at the critical load, Figure 16, as a subtle difference in the shear stress distribution in the lower aft portion of the shell.

This deflection pattern is similar to that seen for the buckling of thin cylinders subject to torsion. ${ }^{14} \mathrm{~A}$ method for designing the structure to prevent this type of failure was investigated. An efficiency equation, similar to those already included in the AXIDES method to design for longitudinal and radial buckling, was found in Reference 15 . For the design of a monocoque shell of intermediate length ( $10 \sqrt{t / R}<L / R<3 \sqrt{R / t})$ subject to torsional buckling:

$$
\frac{N_{x y}}{R E}=.741\left(\frac{t}{R}\right)^{9 / 4}\left(\frac{R}{L}\right)^{1 / 2}
$$

where the critical shear stress is

$$
F_{S_{c r}}=.741 E\left(\frac{t}{R}\right)^{5 / 4}\left(\frac{R}{L}\right)^{1 / 2}
$$

for a simply supported shell. Rearranging Equation 6 to give a relation for the minimum thickness required to prevent torsional buckling for a given shear stress resultant, material, and geometry results in

$$
t_{\text {sbuck }}=R\left[\frac{N_{x y}}{.741 R E}\left(\frac{L}{R}\right)^{1 / 2}\right]^{4 / 9}
$$

Assuming a simply supported shell and analyzing stiffened shells as monocoque with a length equal to the frame spacing, Equation 8 was added to the AXIDES method as an additional failure mode used to compute the smeared shell thickness required at each analysis station. New values of $t_{\min }$ were then computed for both the internal and external cowl structures (Figure 17).

Nonlinear analyses using these new values for $t_{\min }$ were performed for both the internal and external cowl structures. The internal cowl FEA model using the new values for $t_{\min }$ converged to carry 100 percent of the applied load. Figure 18 compares the FEA bending stress from the converged nonlinear analysis with that computed analytically using the new values for $t_{\min }$. The comparison is much better than that resulting from the linear static analysis (Figure 13). The bending stresses exhibit the expected distribution with tensile stresses on the top of the structure, and compressive stresses on the bottom.

Results from the nonlinear analysis of the redesigned external cowl were not as favorable. The structure buckled under only 4.7 percent of the load. The shear stress distribution of the external cowl (Figure 19) shows a different deformation pattern than 
that of the internal cowl (Figure 16). Large

deformations occur at the external cowl lip with virtually no deformation in the aft portion of the shell. The reason for the differences between the internal and external cowl buckling deformation is due to differing load applications. All of the pressures on the internal cowl, normal and hammershock, are applied to the internal surface of the structure. However, due to the difference between the external pressure and the internal leakage pressures, normal and hammershock, the normal pressures in the asymmetric hammershock load case are applied to the external surface of the external cowl, while the hammershock pressures are applied to the internal surface. This results in the large twist evident in Figure 19, causing the external cowl shell to buckle at such a low percentage of the applied load. The external cowl results are discussed further below.

\section{Method Assumptions}

Detail design of an axisymmetric inlet would entail determining the dimensions of each panel, stiffener, frame, actuator, etc., for a specific inlet required to meet specific load conditions. This is called a point design. At the conceptual level of design, performing a point design of an inlet is infeasible as well as impractical. Assumptions are therefore made to simplify the structure for quick analysis. In the development of the AXIDES minimum weight design method, many simplifying assumptions were made. The first concerned the type of construction that would be used to design the inlet. This was assumed to be stiffened shell construction. This limits the application of the method to those inlet types whose structure can reasonably be described using this type of construction. Using AXIDES to design inlets that contain components designed using slats and seals, instead of some type of stiffened panel construction, would produce questionable results. Correction factors could be determined to adjust the weight computed by AXIDES for these types of inlets, however, by comparing with existing inlet data.

The second important assumption, or simplification, made to the inlet involved using smeared structural dimensions instead of discrete values for the design of the shell and longitudinal stiffeners and for the ring frames. As a result, the inlet design produced by AXIDES is not what would ultimately be manufactured. In some cases, the structure designed by AXIDES could not be built. For example, it is highly improbable that a panel would be constructed with a thickness that changes every four inches. This is essentially what is predicted by the analytical method by computing the smeared thickness at analysis stations spaced closer together than the stabilizing frames. However, this simplification makes it possible to consider many variations of an inlet design in a short amount of time, and with little input data.

The structure of the inlet was further simplified for the finite element analyses, where only the smeared shell was modeled, without any stiffeners or frames. Unlike the AXIDES method, which includes analyses based on a stiffened panel and shell structure, and therefore "knows" what the structure really looks like, the finite element methods have no "knowledge" that any stiffening of the structure exists. They "see" the inlet components as no more than large thin shells. This became important for the axisymmetric hammershock load case evaluated above. As discussed, the FEA bending stress for the inlet structures included that due to local deformation of the elements. While this type of deformation might take place in a "real" inlet, stiffeners and supports would be included in a discrete design to ensure it did not lead to structural failure. Since the FEA deformations were small and well below the elastic limit of the material, and considering the simplifications made in the model, the decision was made not to try to correct the AXIDES method for these local deformations.

The third major assumption made in the development of the AXIDES method was that of Classical Beam behavior. The inlet structures were modeled as hollow cylindrical cantilever beams. Since the analysis of these types of beams is relatively simple and well documented, this further enabled the method to produce a minimum weight design quickly and with a small amount of input data. The assumption of beam behavior worked well for the inlet centerbody; however, the FEA deflection and asymmetric pressure load case stress results for the cowl show greater evidence of shell behavior. This is not a surprising result, as the L/D value for the cowl is considerably lower than that given in the literature as the limit for the application of beam theory. It was possible to correct the deflection calculation in AXIDES to account for shell behavior by including the deflection due to shear, as above. It was also possible to better model the internal cowl structure by including an additional failure mode, that of torsional buckling.

The FEA deformation and stresses of the external cowl under asymmetric hammershock loads, however, were not easily modeled by the analytical method. Considering again the assumptions made in the development of the FEA models as compared with the construction of a real inlet, one can see that it is likely 
that the FEA deformations and stresses would not be present in a structure designed using discrete elements. To modify the analytical method to match the current FEA results exactly for this case would not be reasonable. Final evaluation of the AXIDES method will have to be made by comparing the analytical results with real in service inlets, or with study inlets that have been advanced to the detail design phase. This will be the next, and final, step in validating the new analytical minimum weight design method.

\section{Conclusion}

A new method for developing a minimum weight design of a high-speed axisymmetric inlet was demonstrated using a generic Mach 2.4 axisymmetric inlet. The method, AXIDES, uses analytical principles to determine the minimum equivalent isotropic thicknesses of a stiffened shell structure and transverse ring frames. The GENESIS and NASTRAN finite element programs were used to evaluate the deflections and stresses predicted by the AXIDES method.

After modifications were made to include the calculation of shear stresses and a torsional buckling failure mode, the AXIDES method was demonstrated to develop a minimum weight structure that meets all of the defined load requirements with the exception of asymmetric hammershock pressure. The shell behavior of the inlet external cowl model under the asymmetric hammershock loads is not indicative of the behavior of a real inlet built with discrete structural elements. Comparisons with real inlets will be necessary to completely verify the design of the external cowl and determine necessary correction factors to be applied in the AXIDES method.

The final step in evaluating the new AXIDES method will be applying it to existing inlets and comparing the computed weights with actual values. Once this has been completed, the method will be ready to use in systems studies supporting the aeronautics project offices at the National Aeronautics and Space Administration as well as the aeronautics industry.

\section{References}

1. Nadell, S.B.: Axisymmetric Inlet Minimum Weight Design Method. NASA TM-106948, Aug., 1995.

2. Ardema, M.D.: Body Weight of Hypersonic Aircraft: Part 1. NASA TM-101028, Oct., 1988.
3. Seddon, J.; Goldsmith, E.L.: Intake Aerodynamics. AIAA, Inc. 1985.

4. Shanley, F.R.: Weight-Strength Analysis of Aircraft Structures. Dover Publications, Inc. 1960.

5. Kowalski, E.J.: A Computer Code for Estimating Installed Performance of Aircraft Gas Turbine Engines, Vols. I-III. NASA CR159691-3. Dec., 1979.

6. Timoshenko, S.: Strength of Materials Part I. D Van Nostrand Company, Inc., 1955.

7. Military Standardization Handbook. Metallic Materials and Elements for Aerospace Vehicle Design. MIL-HDBK-5E, 1987.

8. Nadell, S.B.: Investigation of Methods for the Structural Weight Analysis of a Mach 2.4 Axisymmetric Inlet. NASA TM-106693, Aug., 1994.

9. Koncsek, J.L.; Gerstle, J.H.; MacKinnon, M.I.K.: Inlet Type Screening for High Speed Civil Transport with Turbine Bypass Engine. The Boeing Commercial Airplane Company. NAS325963. CR101006. Mar., 1993.

10. MSC/PATRAN Release 1.4-I Users Manual. The MacNeal-Schwendler Corporation, Los Angeles, CA.

11. GENESIS Users Manual. VMA Engineering, Colorado Springs, CO.

12. Roarke, R.J.; Young, W.C.: Formulas for Stress and Strain. Fifth Edition. McGraw-Hill, Inc., 1975.

13. NASTRAN Users Manual. The MacNealSchwendler Corporation, Los Angeles, CA.

14. Donnell, L.H.: Beams, Plates, and Shells. McGraw-Hill, Inc., 1976.

15. Crawford, R.F.; Burns, A.B: Strength, Efficiency, and Design Data for Beryllium Structures. N6310645. Feb., 1962. 

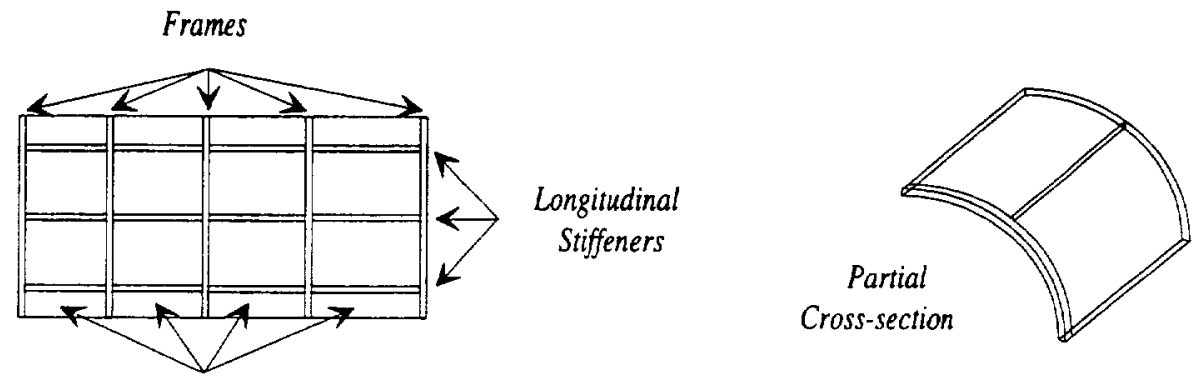

Panels

Figure 1. Stiffened Shell Geometry

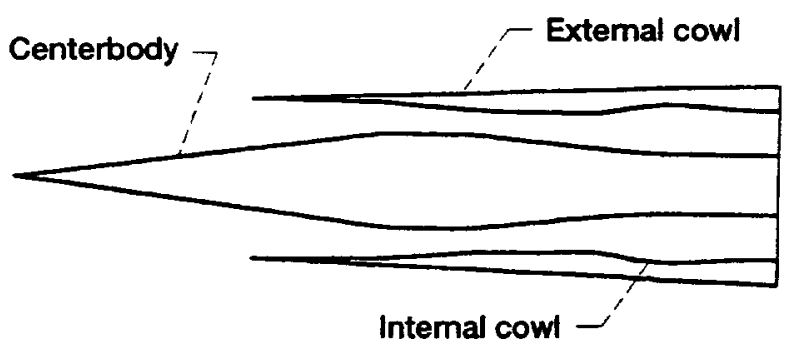

Figure 2. Axisymmetric Inlet Components

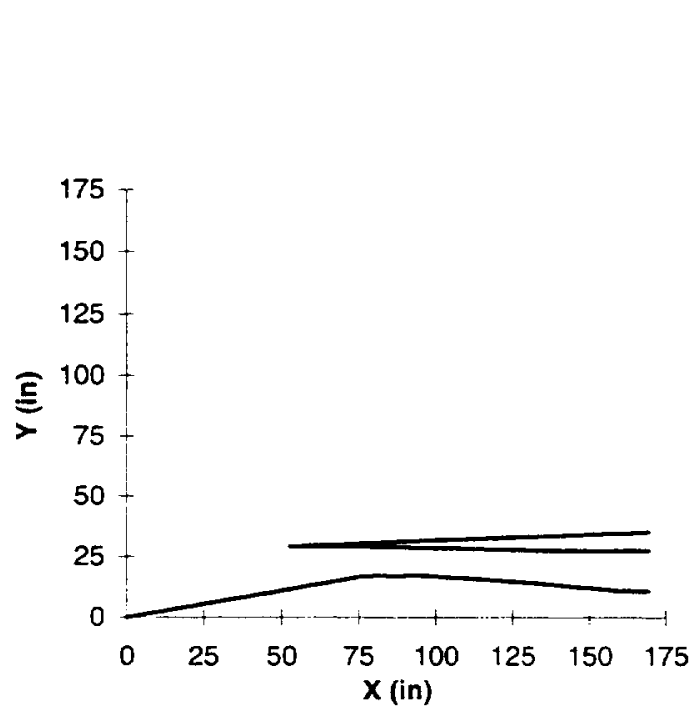

Figure 3. Generic Axisymmetric Inlet Geometry

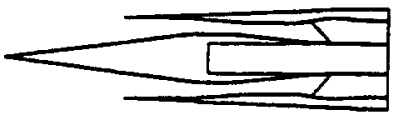

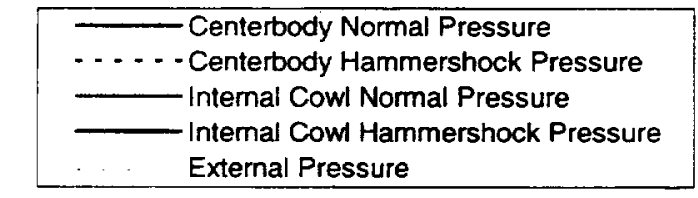

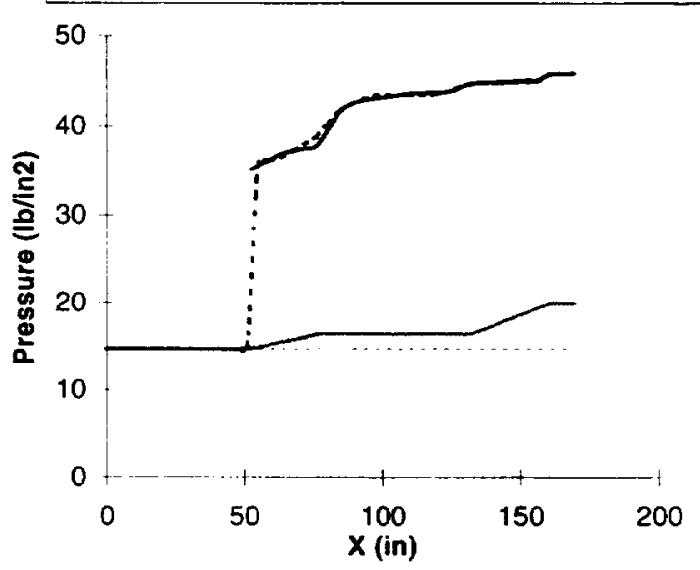

Figure 4. Generic Axisymmetric Inlet Pressures 


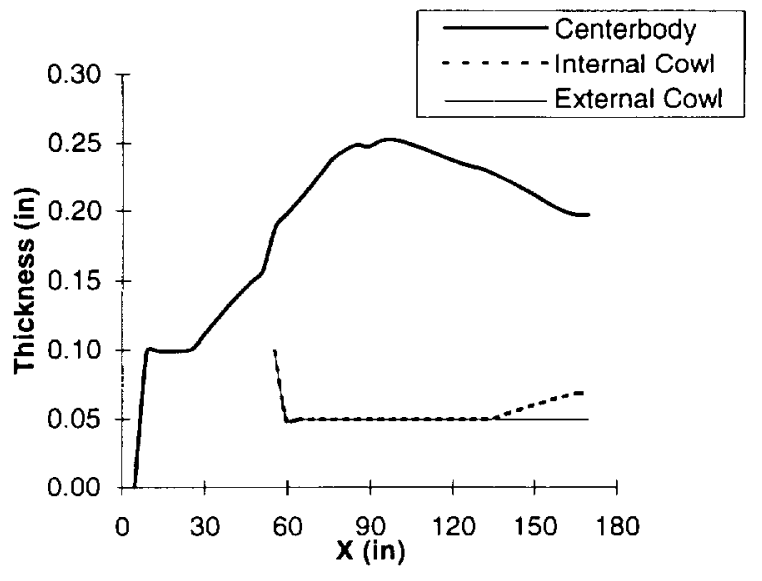

Figure 5. Minimum Equivalent Isotropic Shell Thickness
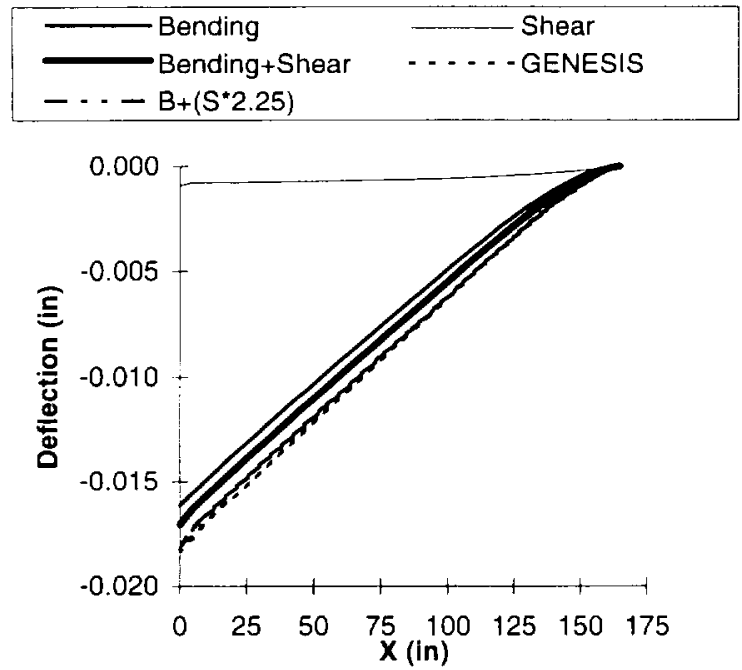

Figure 7. Centerbody Deflection

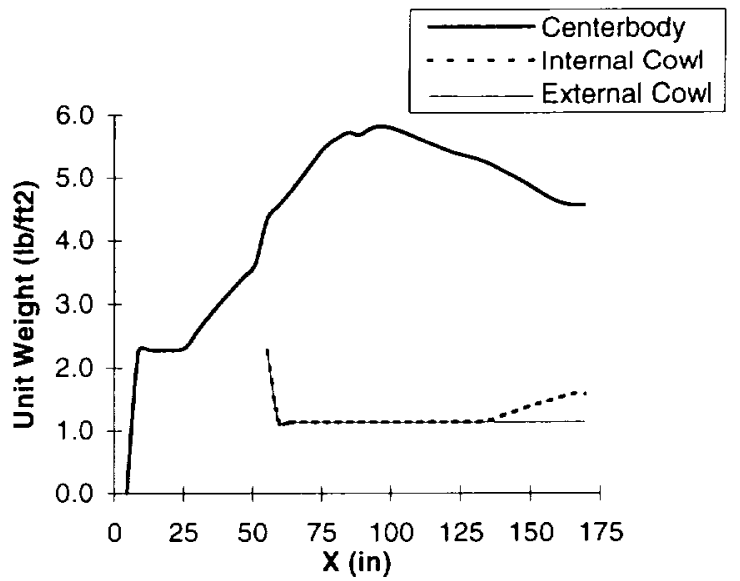

Figure 6. Generic Axisymmetric Inlet Unit Weight

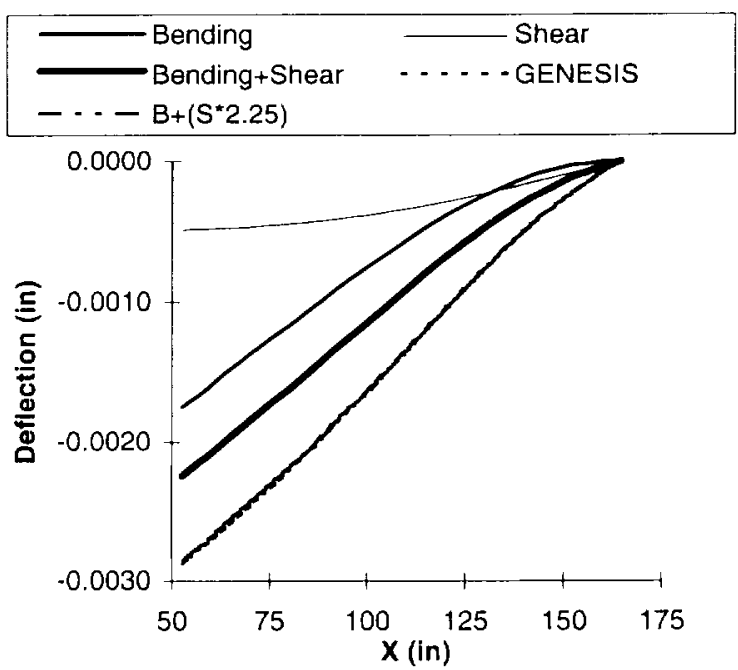

Figure 8. Internal Cowl Deflection

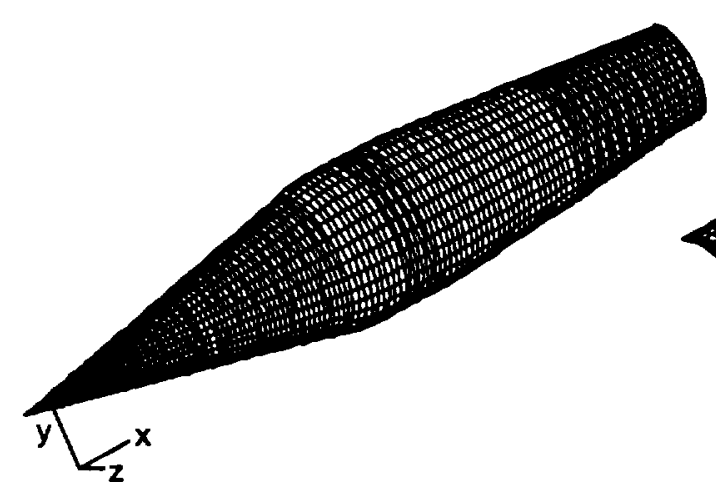

Centerbody

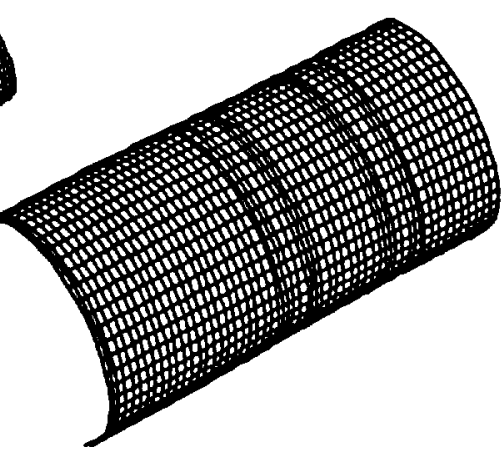

Internal Cowl

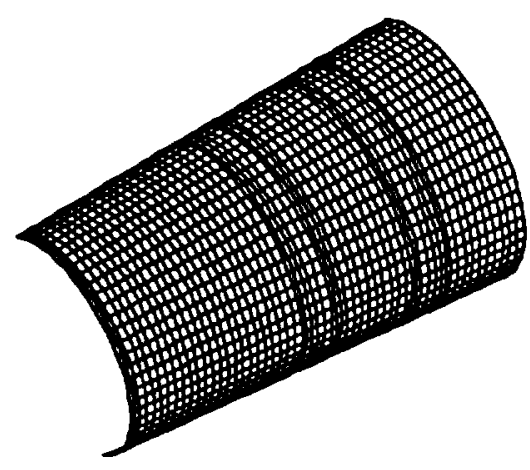

External Cowl

Figure 9. Inlet Finite Element Models 


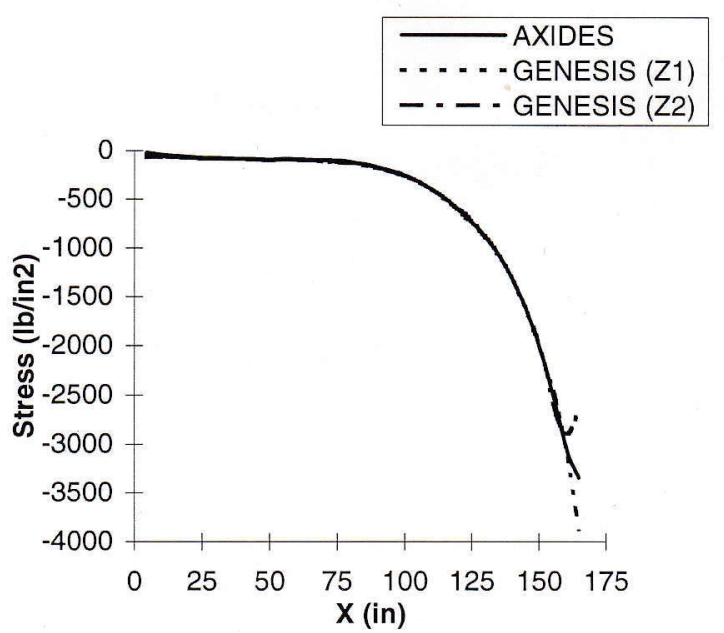

Figure 10. Centerbody Landing Load Case Bending Stress

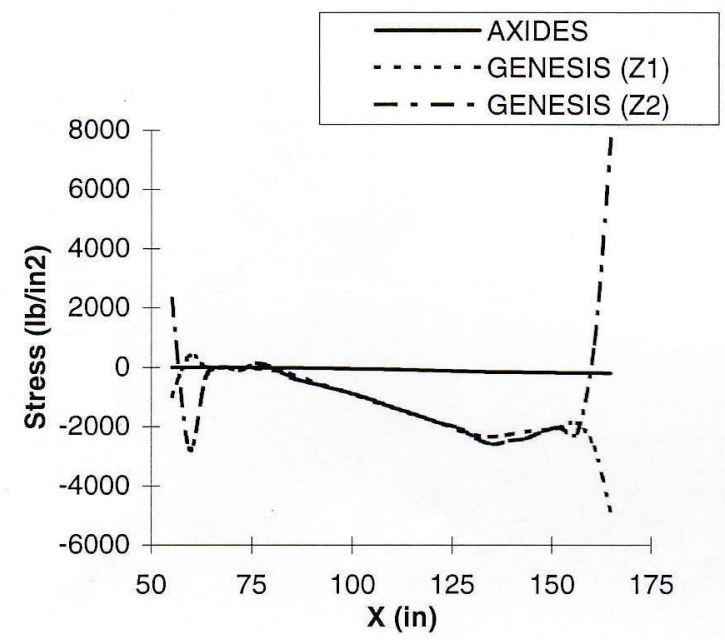

Figure 12. Internal Cowl Axisymmetric Hammershock Load Case Bending Stress

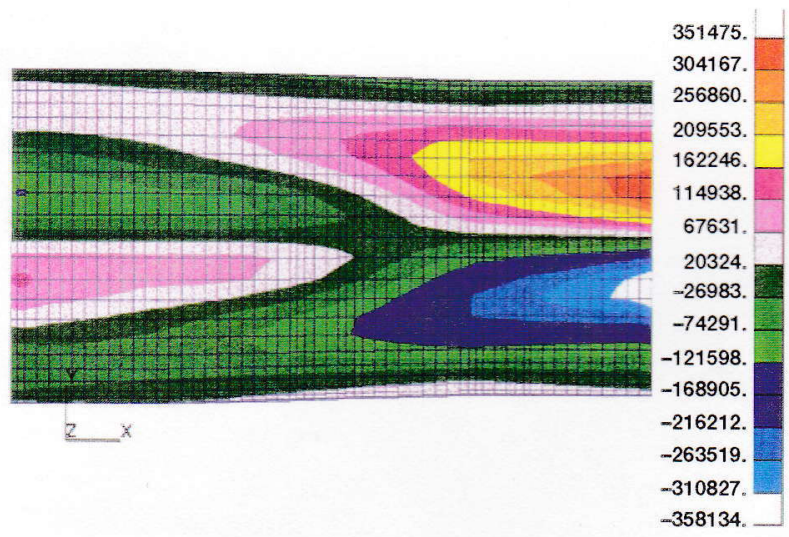

Figure 14. Internal Cowl Asymmetric Hammershock Load Case Bending Stress Distribution

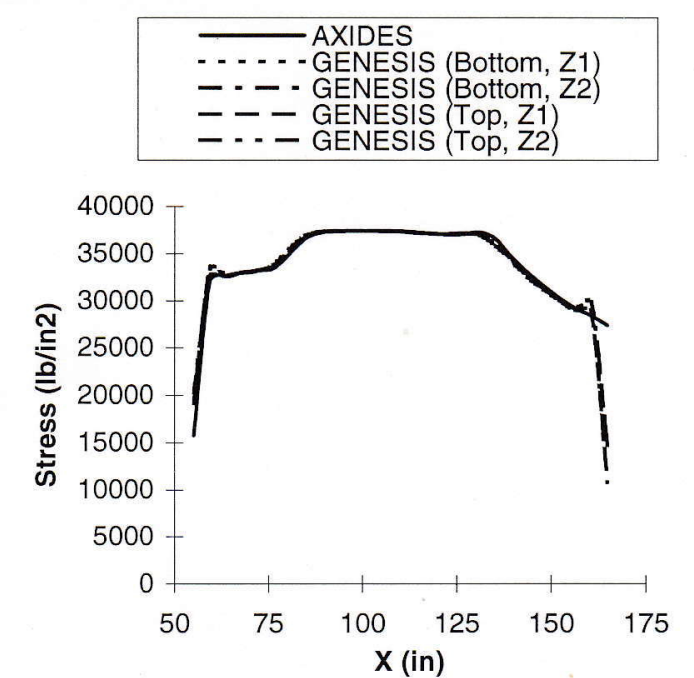

Figure 11. Internal Cowl Axisymmetric Hammershock Load Case Hoop Stress

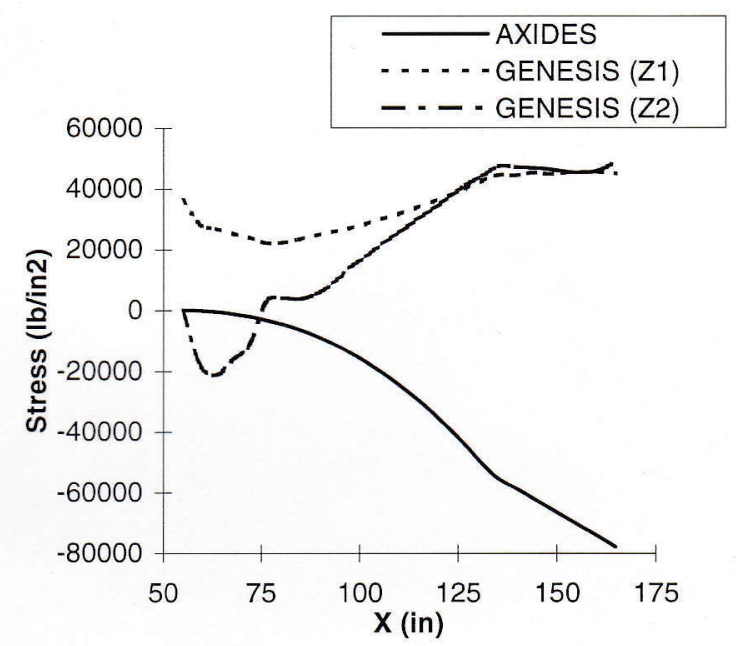

Figure 13. Internal Cowl Asymmetric Hammershock Load Case Bending Stress

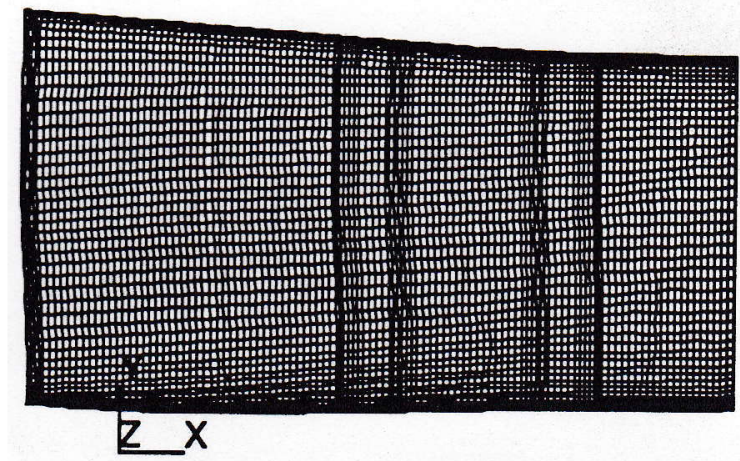

Figure 15. Internal Cowl Buckling Deformation At Critical Load 


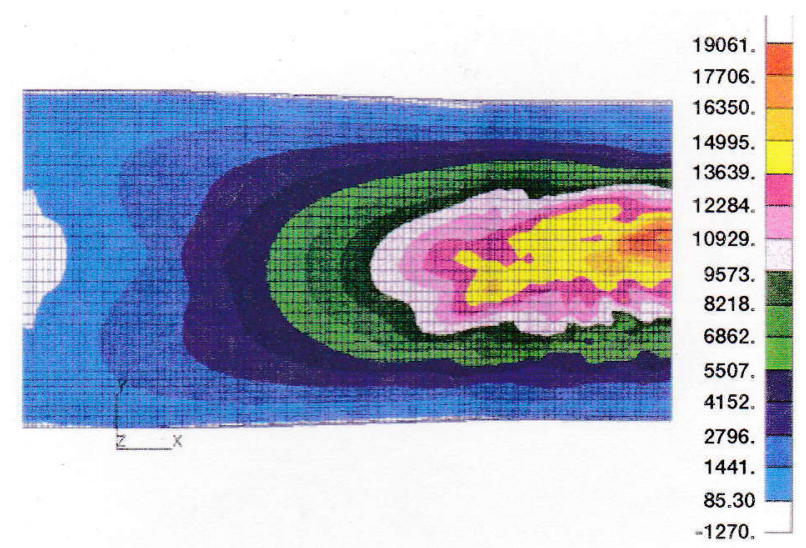

Figure 16. Internal Cowl Shear Stress At Buckling Limit Load

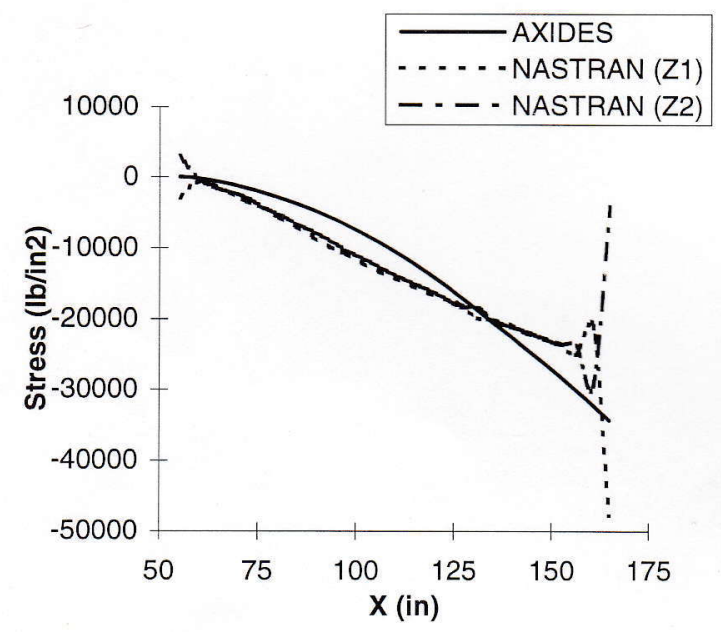

Figure 18. Internal Cowl Bending Stress Using Design For Torsional Buckling

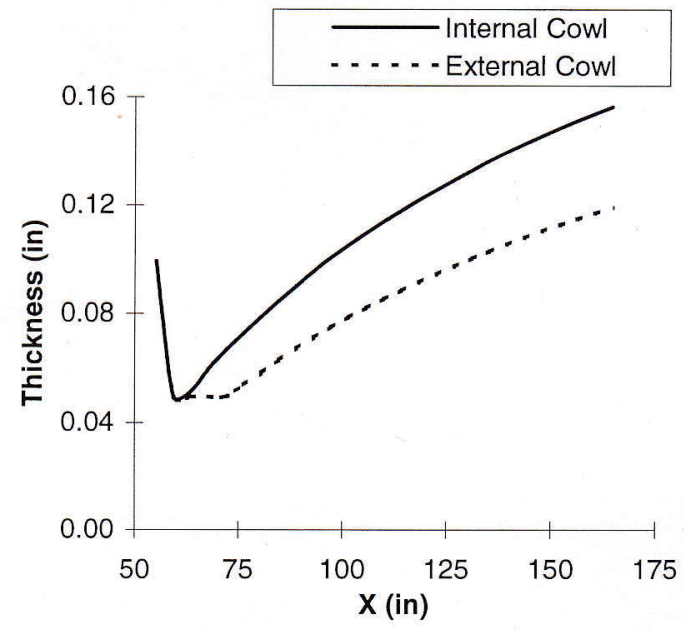

Figure 17. Internal and External Cowl $t_{\min }$ Including Design For Torsional Buckling

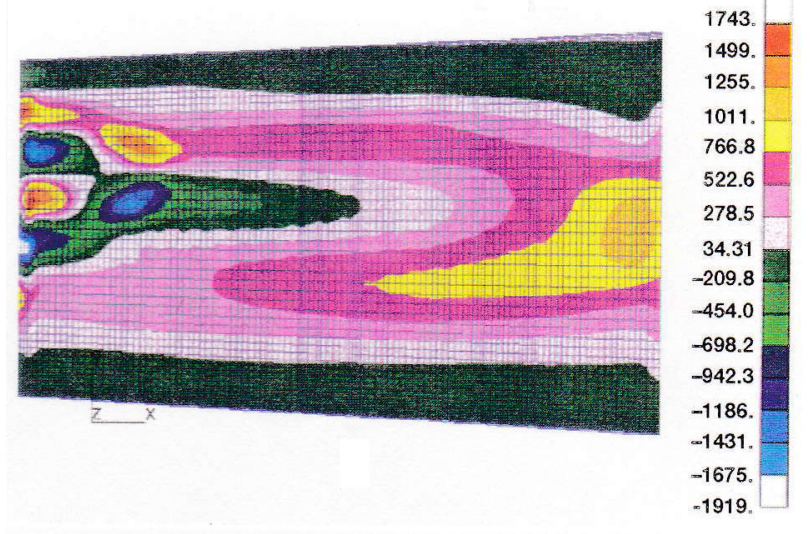

Figure 19. External Cowl Critical Shear Stress Distribution 


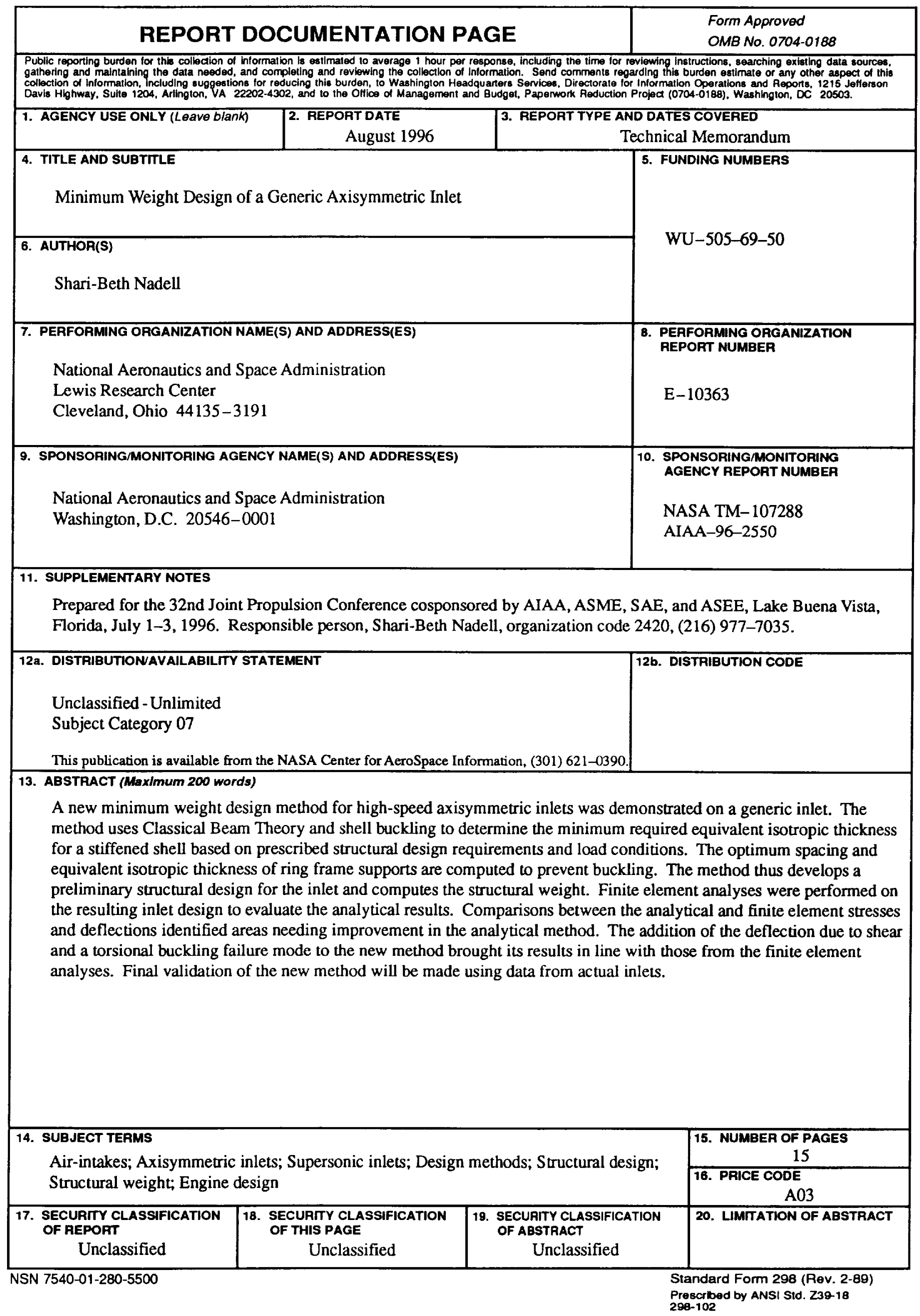




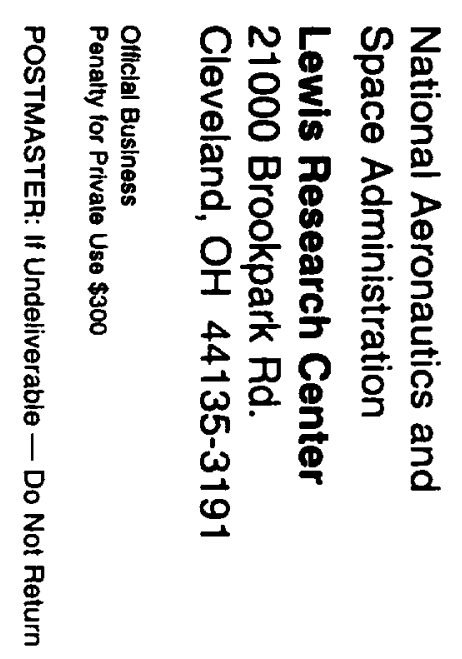

